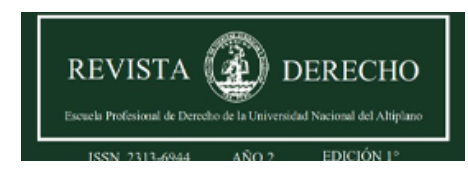

Revista de Derecho

ISSN: 2313-6944

ISSN: 2707-9651

revistaderecho@unap.edu.pe

Universidad Nacional del Altiplano

Perú

\title{
El cambio de línea jurisprudencial en el acceso a la pensión de viudez a raíz de la sentencia recaída en el Exp. $N^{\circ}$ 00617-2017-PA/TC del Tribunal Constitucional
}

\author{
Yabar Juli, Ibet \\ El cambio de línea jurisprudencial en el acceso a la pensión de viudez a raíz de la sentencia recaída en el Exp. N \\ - 00617-2017-PA/TC del Tribunal Constitucional \\ Revista de Derecho, vol. 5, núm. 2, 2020 \\ Universidad Nacional del Altiplano, Perú \\ Disponible en: https://www.redalyc.org/articulo.oa?id=671870937011 \\ DOI: https://doi.org/10.47712/rd.2020.v5i2.93
}

\section{(c) (1)}

Esta obra está bajo una Licencia Creative Commons Atribución 4.0 Internacional. 
Artículos de naturaleza variada

\title{
El cambio de línea jurisprudencial en el acceso a la pensión de viudez a raíz de la sentencia recaída en el Exp. $\mathrm{N}^{\circ}$ 00617-2017-PA/TC del Tribunal Constitucional
}

\begin{abstract}
The change of jurisprudential line in the access to the widow's pension as a result of the sentence in the Exp. $\mathrm{N}^{\circ}$ 00617-2017-PA / TC of the Constitutional Court
\end{abstract}

Ibet Yabar Juli

Corte Superior de Justicia de Puno, Perú

(D) https://orcid.org/0000-0003-2189-7251

DOI: https://doi.org/10.47712/rd.2020.v5i2.93

Redalyc: https://www.redalyc.org/articulo.oa? id $=671870937011$

Recepción: 30 Septiembre 2020

Aprobación: 25 Octubre 2020

Publicación: 27 Octubre 2020

\section{RESUMEN:}

El artículo $53^{\circ}$ del Decreto Ley $N^{\circ} 19990$ del Sistema Nacional de Pensiones, prevé el derecho a la pensión de viudez, al que tiene derecho el cónyuge del asegurado o pensionista fallecido, otorgado a las viudas por dicha condición, en cambio, a los viudos se les exige que cumplan requisitos adicionales como que haya sido dependiente de la viuda, entre otros. Esa situación, a juicio de la autora, vulnera el derecho a la igualdad, en su manifestación de igualdad en la ley porque hace una diferenciación injustificada entre varones y mujeres, ya que no acceden a la pensión en igualdad de condiciones, además, el Tribunal Constitucional hasta hace unos meses atrás desestimaba demandas de amparo donde los viudos solicitaban acceder a la pensión aplicando el artículo $53^{\circ}$, sin embargo, recientemente dicha corporación cambió de criterio tal como se demuestra en el Exp. №06617-2017-PA/TC, ya que declara fundada la demanda e inaplica el artículo $53^{\circ}$ del Decreto Ley $\mathrm{N}^{\circ} 19990$ por vulnerar el derecho a la igualdad, a la vez, declara estado de cosas inconstitucional en relación al tratamiento legislativo desigual por sexo respecto de los requisitos para obtener pensión de viudez.

Palabras Clave: Igualdad, línea jurisprudencial, pensiones, Tribunal Constitucional.

\section{ABstract:}

Article 53 of Decree-Law No. 19990 of the National Pension System, to stablish the right to a widow's pension, the spouse of the insured or deceased pensioner is entitled, granted to widows for said condition, instead, to the Widowers are required to meet additional requirements such as having been a dependent of the widow, among others. This situation, in the author's opinion, violates the right to equality, in its manifestation of equality in the law because it makes an unjustified differentiation between men and women, since they do not have access to the pension under equal conditions, in addition, the Court Constitutional until a few months ago it dismissed claims for protection where widowers requested access to the pension applying article $53^{\circ}$, however, recently the corporation changed its criteria as demonstrated in Exp. No. 00617-2017-PA / TC, since it declares the claim founded and disapplies Article 53 of Decree Law No. 19990 for violating the right to Equality, at the same time, declares an unconstitutional state of affairs in relation to the unequal legislative treatment by sex with respect to the requirements to obtain a widow's pension. Keywords: Equality, jurisprudential line, pensions, Constitutional Court.

KeYwords: Equality, jurisprudential line, pensions, Constitutional Court.

\section{INTRODUCCIÓN}

Este trabajo se justifica a raíz de una experiencia personal -para la autora del presente artículo-, pues el año 2011 fallece mi madre, maestra de educación secundaria, aportante a la Oficina de Normalización Previsional-ONP, quedando viudo mi padre, quien también es profesor, en la actualidad cesante, aportante al mismo sistema pensionario, sin embargo, su condición de viudo no era suficiente para acceder a la pensión de viudez, conforme a lo previsto en el artículo $53^{\circ}$ del Decreto Ley $\mathrm{N}^{\circ} 19990$.

El Tribunal Constitucional en su línea jurisprudencial sobre la materia, en diversos procesos de amparo sostenía que las demandas de viudos que no cumplían los requisitos adicionales previstos por el artículo $53^{\circ}$ 
del Decreto Ley $\mathrm{N}^{\circ} 19990$-el cónyuge inválido o mayor de sesenta años de la asegurada o pensionista fallecida que haya estado a cargo de ésta- fueron desestimados. Tal interpretación y construcción jurisprudencial era contraria a los derechos fundamentales, en este caso, el derecho a la igualdad en la ley, es así que el presente año emite sentencia en el Exp. $\mathrm{N}^{\circ}$ 00617-2017-PA/TC declarando fundada la demanda, apartándose de criterios anteriores, lo que en gran medida significa que reconoce la desigualdad existente en el acceso a la pensión de viudez con relación a los viudos, además declara inaplicable el artículo $53^{\circ}$, a su vez, ve la necesidad de indicar la existencia de un estado de cosas inconstitucional en relación al tratamiento legislativo desigual en razón del sexo a los requisitos para obtener pensión de viudez, ello para que el Poder Legislativo corrija dicho estado.

Lo que consideramos con relación a la sentencia recaída en el Exp. $\mathrm{N}^{\circ}$ 00617-2017-PA/TC es que garantiza el derecho a la igualdad de los pensionistas tanto mujeres y varones. El cambio o variación de criterio jurisprudencial es saludable porque permite a los viudos y viudas acceder a la pensión en igualdad de condiciones, en cambio, anteriormente la línea jurisprudencial que había contraído el Tribunal Constitucional es justificar la desigualdad entre varones y mujeres, situación que agraviaba derechos fundamentales. En un contexto de crisis, inestabilidad e incertidumbre el acceso a la pensión debe darse en igualdad de condiciones, ya que es un aporte que efectuaron de manera legítima y válida durante varios años los asegurados o pensionistas, entonces, era inválido que existieran barreras para hacer diferenciaciones en el acceso a dicho derecho.

Finalmente, debemos resaltar la decisión del Tribunal Constitucional recaída en el Exp. № 00617-2017$\mathrm{PA} / \mathrm{TC}$ porque ahora los viudos podrán acceder a la pensión de viudez por fallecimiento de la cónyuge y con ello mejorar su calidad de vida, además, es una forma de hacer justicia porque tanto el varón como la mujer aportaron en las mismas condiciones al sistema de pensiones y, por supuesto, compartieron los deberes maritales y hubo asistencia recíproca. Entonces, teniendo en cuenta las condiciones previas, este trabajo analiza dos aspectos: (i) vulneración de derechos por el tratamiento diferenciado existente en el artículo $53^{\circ}$ y (ii) impacto de la sentencia del Tribunal Constitucional que cambia el criterio jurisprudencial.

\section{Materiales Y MÉTOdos}

El método empleado es el cualitativo porque busca comprender el problema de la vulneración de derechos fundamentales, además, interpreta la jurisprudencia del Tribunal Constitucional para aclarar las dimensiones o ámbitos que resultaron incompatibles con los derechos. La técnica es el análisis documental porque, esencialmente, se trabaja con sentencias del Tribunal Constitucional y doctrina constitucional vinculado con el derecho a la pensión, lo cual supone evaluar el contenido de cada documento. En este caso, el problema que se aborda que consiste en el cumplimiento de requisitos adicionales para los varones viudos, a diferencia de las viudas, a pesar de que los aportes se dieron en igualdad de condiciones, están contenidos en documentos, esto es, Constitución, leyes y sentencias del Tribunal Constitucional.

\section{LA SEGURIDAD SOCIAL Y SISTEMAS DE PENSIONES EN LA JURISPRUDENCIA DEL TRIBUNAL Constitudional}

El artículo $10^{\circ}$ de la Constitución reconoce y garantiza el derecho universal y progresivo de toda persona a la seguridad social, que en materia de pensiones tiene una doble finalidad, por un lado, proteger a la persona frente a las contingencias derivadas de la vejez, la invalidez o la muerte; y, por otro, elevar su calidad de vida, lo cual se concreta a través de las prestaciones que brindan los distintos regímenes de pensiones establecidos por el legislador (Vidal, 2015). En la misma línea, el artículo $11^{\circ}$ de la Constitución establece que "El Estado garantiza el libre acceso a prestaciones de (...) pensiones, a través de entidades públicas, privadas o mixtas. Supervisa asimismo su eficaz funcionamiento". Con lo que, en materia de pensiones, el constituyente ha 
reconocido que las prestaciones pueden ser otorgadas mediante entidades públicas, privadas y/o mixtas, poniendo en relieve que el Estado asume un rol supervisor y/o fiscalizador cuando las prestaciones sean brindadas por entidades privadas y/o mixtas (Clark, 2009).

Teniendo en cuenta esos aspectos, el Tribunal Constitucional, indicó que la seguridad social y el libre acceso a las prestaciones pensionarias tienen que ser considerados como derechos de configuración legal porque a través de la ley se precisan los requisitos y condiciones que se deben cumplir a efectos de acceder y gozar de las prestaciones que cada uno de los regímenes pensionarios establece en cada caso en particular. También hubo oportunidad para precisar que el país cuenta con dos sistemas de pensiones que coexisten, el Sistema Público de Pensiones, que agrupa más de un régimen de reparto, como son los establecidos por los Decretos Leyes Nos 19846, 19990 y 20530, entre otros; y por el otro, el Sistema Privado de Pensiones, que es un único régimen de capitalización individual creado por el Decreto Ley No 25987 (EXP. N 1417-2005$\mathrm{AA} / \mathrm{TC})$.

De este modo, le compete al legislador establecer los mecanismos de acceso al sistema, el conjunto de prestaciones y los requisitos para acceder a los mismos, así como los esquemas de financiamiento de los sistemas de pensiones. Obviamente que al momento de configurar los sistemas de pensiones el legislador tiene como límites los derechos fundamentales a la dignidad humana, a la vida, a la seguridad social y al libre acceso a las prestaciones pensionarias, así como los principios de universalidad, solidaridad y progresividad (Gonzales, 2017).

IV. El desarrollo jurisprudencial del Tribunal Constitucional sobre el derecho A LA PENSIÓN: ANÁLISIS ESTRICTO DESDE LA PERSPECTIVA DE LA LÍNEA JURISPRUDENCIAL CONSTRUIDA POR EL MÁXIMO INTÉRPRETE DE LA CONSTITUCIÓN

En los últimos años, el Tribunal Constitucional ha venido incrementando su producción jurisprudencial, en especial, logrando emitir sentencias con calidad de precedente que instauraron nuevas reglas en el sistema jurídico. Ese tipo de decisiones fueron emitidas en diversas materias incluido el derecho a la pensión y que son reglas de aplicación obligatoria porque todos los jueces de la república quedan obligados a cumplir dichas reglas. 


\begin{tabular}{|l|l|l|}
\hline Expediente & Caso & Materia regulada \\
\hline $\begin{array}{l}\text { STC } \\
\text { 02677-2016-PA/TC }\end{array}$ & $\begin{array}{l}\text { Ladislao } \\
\text { Carrillo Espejo }\end{array}$ & $\begin{array}{l}\text { Pagos en exceso al } \\
\text { pensionista. }\end{array}$ \\
\hline $\begin{array}{l}\text { STC } \\
\text { 00799-2014-PA/TC }\end{array}$ & $\begin{array}{l}\text { Mario Eulogio } \\
\text { Flores Callo }\end{array}$ & $\begin{array}{l}\text { Validez de los certificados } \\
\text { médicos en materia } \\
\text { pensionaria. }\end{array}$ \\
\hline $\begin{array}{l}\text { STC } \\
\text { 02513-2007-PA/TC }\end{array}$ & $\begin{array}{l}\text { Ernesto } \\
\text { Casimiro } \\
\text { Hernández } \\
\text { Hernández }\end{array}$ & $\begin{array}{l}\text { Pensión vitalicia y pensión } \\
\text { de invalidez. }\end{array}$ \\
\hline $\begin{array}{l}\text { STC } \\
\text { 04762-2007-PA/TC }\end{array}$ & $\begin{array}{l}\text { Alejandro } \\
\text { Tarazona } \\
\text { Valverde }\end{array}$ & $\begin{array}{l}\text { Acreditación de } \\
\text { aportaciones en materia } \\
\text { pensionaria. }\end{array}$ \\
\hline $\begin{array}{l}\text { STC } \\
\text { 05430-2006-PA/TC }\end{array}$ & $\begin{array}{l}\text { Alfredo de la } \\
\text { Cruz Curasma }\end{array}$ & $\begin{array}{l}\text { Pago de devengadose } \\
\text { intereses en materia } \\
\text { pensionaria. }\end{array}$ \\
\hline $\begin{array}{l}\text { STC } \\
\text { 06612-2005-PA/TC }\end{array}$ & $\begin{array}{l}\text { Onofre } \\
\text { Vilcarima } \\
\text { Palomino }\end{array}$ & $\begin{array}{l}\text { Pensión vitalicia y pensión } \\
\text { de invalidez; enfermedad } \\
\text { profesional. }\end{array}$ \\
\hline $\begin{array}{l}\text { STC } \\
10087-2005-P A / T C\end{array}$ & $\begin{array}{l}\text { Alipio Landa } \\
\text { Herrera }\end{array}$ & $\begin{array}{l}\text { Decreto Ley 18846, Ley } \\
\text { 2679o, pensión vitalicia y } \\
\text { pensión de invalidez; } \\
\text { enfermedad profesional }\end{array}$ \\
\hline $\begin{array}{l}\text { STC } \\
\text { 05189-2005-PA/TC }\end{array}$ & $\begin{array}{l}\text { Jacinto Gabriel } \\
\text { Angulo }\end{array}$ & $\begin{array}{l}\text { Aplicación de la Ley } \\
\text { 239o8, pensión mínima o } \\
\text { inicial. }\end{array}$ \\
\hline $\begin{array}{l}\text { STC } \\
\text { 01417-2005-PA/TC }\end{array}$ & $\begin{array}{l}\text { Manuel } \\
\text { Anicama } \\
\text { Hernández }\end{array}$ & $\begin{array}{l}\text { Amparo provisional; } \\
\text { contenido esencial del } \\
\text { derecho a la pensión. }\end{array}$ \\
\hline
\end{tabular}

Estas decisiones resaltan la importancia del derecho a la pensión y su necesidad de protección, abriendo verdaderas líneas jurisprudenciales que han servido para reconocer el derecho a la pensión a las personas. También desde el Tribunal Constitucional, se impulsó la necesidad de acceder a la pensión de viudez en igualdad de condiciones, esto es, la proscripción de las desigualdades existentes con relación a dicho derecho, sin embargo, tal como exponemos en este trabajo notamos que todavía existen brechas y problemas con relación a la materialización de dicho derecho. Así tenemos que mediante la sentencia del Tribunal Constitucional recaída en el Exp. $\mathrm{N}^{\circ}$ 00617-2017-PA/TC se reconoce la existencia de desigualdad, pero que fue resuelto por esa sentencia. Entonces, lo que eso evidencia es que el acceso al derecho a la pensión en igualdad de condiciones no estaba vigente ni resultaba aplicable para los viudos.

Por otro lado, cabe indicar que el derecho fundamental a la pensión tiene un contenido esencial que garantizar, ya que tiene relevancia para la protección del adulto mayor, siendo así, el proceso constitucional de amparo es la vía fundamental para garantizar la protección del derecho fundamental a la pensión. El contenido esencial de dicho derecho se traduce en las siguientes dimensiones: (i) disposiciones legales que establecen los requisitos del libre acceso al sistema de seguridad social consustanciales a la actividad laboral pública o privada, dependiente o independiente, y que permite dar inicio al período de aportaciones al Sistema Nacional de Pensiones; por ello, son objeto de protección por vía del amparo los supuestos en los que habiendo el demandante cumplido dichos requisitos legales se le niegue el acceso al sistema de seguridad social; (ii) disposiciones legales que establecen los requisitos para la obtención de un derecho a la pensión, también es objeto de protección en la vía de amparo, (iii) derecho fundamental a la pensión tiene una estrecha relación con el derecho a una vida acorde con el principio-derecho de dignidad, es decir, con la trascendencia vital propia de una dimensión sustancial de la vida, antes que una dimensión meramente existencial o formal, forman parte de su contenido esencial aquellas pretensiones mediante las cuales se busque preservar el derecho concreto a un 'mínimo vital', (iv) susceptible de protección a través del amparo los supuestos en los que se deniegue el otorgamiento de una pensión de sobrevivencia, a pesar de cumplir 
con los requisitos legales para obtenerla, (v) titularidad del derecho subjetivo concreto de que se trate debe encontrarse suficientemente acreditada y (vi) disposiciones legales referidas al reajuste pensionario o a la estipulación de un concreto tope máximo a las pensiones (Morales, 2016).

Con lo indicando queda claro que la pensión se rige por el principio de solidaridad que se traduce en una manifestación por erradicar las desigualdades sociales. Eso para reducir significativamente los niveles de pobreza, contribuyendo a que tanto hombres como mujeres de distintas procedencias socio-culturales y raciales puedan acceder en iguales condiciones, pese a haber vivido en circunstancias distintas, a un mínimo que les permite vivir dignamente en su vejez (Vidal, 2015). La pensión debe tender a ser universal, finalidad a la que las políticas públicas deben encaminarse progresivamente (Neves, 2006). En esa línea, de conformidad con los pronunciamientos del Tribunal sobre el acceso a la pensión, es constitucionalmente admisible que la pensión, pese a ser universal, tenga parámetros configurados legalmente, en función a la disponibilidad económica del sistema de seguridad social y al principio de solidaridad (Exp. N. ${ }^{\circ}$ STC 0050-2004-AI y acumulados), sin embargo, lo que no se acepta es que exista desigualdad o tratamiento diferenciado entre varones y mujeres en el acceso a la pensión, ya que si se ha establecido el contenido esencial del derecho a la pensión es para que todos puedan acceder al mismo bajo determinadas circunstancias, siempre que no evidencie diferenciación.

\section{LA igualdad EN EL Sistema de PEnsiones: Jurisprudencia Del Tribunal Constitucional sobre el Artículo 53º Del Decreto Ley Nº 19990}

El artículo 53 del Decreto Ley N 19990 , prevé: “Artículo 53.- Tiene derecho a pensión de viudez la cónyuge del asegurado o pensionista fallecido, y el cónyuge inválido o mayor de sesenta años de la asegurada o pensionista fallecida que haya estado a cargo de ésta, siempre que el matrimonio se hubiera celebrado por lo menos un año antes del fallecimiento del causante y antes de que éste cumpla sesenta años de edad si fuese hombre o cincuenta años si fuese mujer, o más de dos años antes del fallecimiento del causante en caso de haberse celebrado el matrimonio a edad mayor de las indicadas", primer párrafo modificado por el Artículo $2^{\circ}$ de la Ley N³0907, publicada el 11 enero 2019 (tiene derecho no sólo el cónyuge sino también el integrante de la unión de hecho).

Se advierte que con la modificación del primer párrafo del artículo referido, tiene derecho a pensión ya no sólo el cónyuge del asegurado o pensionista fallecido, sino también el integrante sobreviviente de la unión de hecho.

También se desprende que el legislador otorga un tratamiento diferenciado para la obtención de pensión de viudez, entre viudas y viudos, como se aprecia en el siguiente gráfico: 


\begin{tabular}{|c|c|c|}
\hline \multicolumn{3}{|l|}{ PENSIÓN DE VIUDEZ } \\
\hline \multicolumn{3}{|c|}{ Decreto Ley 19990 (Artículo 53) } \\
\hline $\begin{array}{l}\text { CRITERIOS DE } \\
\text { DIFERENCIACIÓN }\end{array}$ & MUJERES & VARONES \\
\hline Condición de salud. & Sana & $\begin{array}{l}\text { Inválido (sano, solo } \\
\text { si es mayor de } 60 \\
\text { años de edad). }\end{array}$ \\
\hline $\begin{array}{l}\text { Edad mínima para } \\
\text { obtener la pensión de } \\
\text { viudez. }\end{array}$ & $\begin{array}{l}\text { No hay edad } \\
\text { minima }\end{array}$ & $\begin{array}{l}60 \text { años (si no es } \\
\text { inválido) }\end{array}$ \\
\hline $\begin{array}{l}\text { Edad máxima del } \\
\text { cónyuge a la fecha de } \\
\text { celebración del } \\
\text { matrimonio o de la } \\
\text { unión de hecho. }\end{array}$ & 60 años & 50 años \\
\hline $\begin{array}{l}\text { Dependencia económica } \\
\text { del causante. }\end{array}$ & NO & SI \\
\hline
\end{tabular}

Gráfico del fundamento de voto del magistrado Espinosa-Saldaña Barreda Exp. Nº0617-2017-PA/TC.

Es así, que en la jurisprudencia del Tribunal Constitucional, las demandas constitucionales de amparo, interpuestas por viudos que pretendían se les otorgue pensión de viudez en aplicación del artículo $53^{\circ}$ del Decreto Ley $\mathrm{N}^{\circ}$ 19990, han sido desestimadas, como se detalla: Exp. $\mathrm{N}^{\circ} 00313-2010-\mathrm{PA} / \mathrm{TC}$ : "el actor no ha demostrado que se haya encontrado a cargo de su cónyuge" (Fj.4); Exp. No02380-2010-PA/TC: "No obstante ello, el actor no ha demostrado fehacientemente que se haya encontrado a cargo de su cónyuge" ( $\mathrm{Fj}$. 5); Exp. N04045-2016-PA/TC, se emitió sentencia interlocutoria, señalando: "este Tribunal, en reiterada jurisprudencia, ha señalado que en el caso de la pensión de viudez del cónyuge varón, se busca proteger el estado de desamparo en que pudiera quedar el cónyuge del beneficiario de una pensión al no encontrarse en condiciones de atender su subsistencia por sus propios medios. En el caso de autos, de la boleta de fojas 2 se advierte que el recurrente percibe una pensión de jubilación conforme al Decreto Ley 19990 por la suma de S/.462.94, monto que resulta superior a media remuneración vital. Por tanto, no encontrándose dentro de los supuestos establecidos en el artículo 53 del Decreto Ley 19990, no le corresponde acceder a la pensión de viudez solicitada".

Del contexto descrito se advierte, que el Tribunal Constitucional, no realizaba mayor análisis, que verificar el cumplimiento de los requisitos previstos en el artículo 53 del Decreto Ley $\mathrm{N}^{\circ}$ 19990; empero, en la sentencia contenida en el Exp. No0617-2017-PA/TC de fecha 18 de noviembre de 2019 (Sentencia 25/2020), aplica el test de igualdad (Exp. Nº0045-2004-AI/TC), para verificar la constitucionalidad del citado artículo, concluyendo que la norma es lesiva de derechos fundamentales y su aplicación desconoce la supremacía de la Constitución (Fj.35), por lo que se aparta de los criterios anteriores, porque se atenta contra el derecho a la igualdad en la ley (Fj.37).

También se desprende que en la referida sentencia, donde el Tribunal cambia de criterio, el demandante tiene la condición de jubilado, y en la absolución de la demanda la ONP señaló no sólo el incumplimiento de los requisitos previstos en el artículo $53^{\circ}$ del Decreto Ley $\mathrm{N}^{\circ} 19990$, sino también refiere que el demandante percibe una pensión por derecho propio, empero se desprende que si bien el Tribunal no mite pronunciamiento en dicho extremo, se colige que las pensiones son de naturaleza distinta y no serían incompatibles por su naturaleza y su origen; pues finalmente, el Tribunal no sólo estima la demanda, declarando inaplicable el citado artículo, sino también declara el estado de cosas inconstitucional, técnica mediante la cual se tutela derechos fundamentales cuando los afectados son un grupo de personas.

Por último, categóricamente, sostenemos que el cambio de criterio jurisprudencial del Tribunal Constitucional es positivo porque garantiza que la igualdad en el acceso a pensión tanto de mujeres y varones, situación que con la construcción jurisprudencial -previa a la sentencia recaída en el Exp. N. ${ }^{\circ}$ 00617-2017- 
PA/TC- no sucedía. En ese orden, estamos de acuerdo con que el máximo intérprete de la Constitución haya cambiado el modo de analizar y enfocar el artículo $53^{\circ}$ advirtiendo que generaba desigualdad entre varones y mujeres. Felizmente, esa situación ha variado y ahora el viudo puede acceder y gozar de la pensión de viudez en las mismas condiciones que la mujer, esto es, el esposo viudo justificadamente se beneficia de los esfuerzos y el fruto del trabajo de su esposa.

\section{Conclusión}

El cambio de criterio asumido por el Tribunal Constitucional Exp. N. ${ }^{\circ} 00617-2017-\mathrm{PA} / \mathrm{TC}$ es justificado porque anteriormente la línea jurisprudencial que se había construido generaba desigualdad en el acceso a la pensión entre varones y mujeres, ya que el artículo $53^{\circ}$ exigía que el varón para acceder a la pensión de la viudez por fallecimiento de la cónyuge tenía que acreditar que dependía de esta o era inválido, entre otros. Esa situación ha cambiado con la sentencia recaída en el expediente que es materia de reflexión en este trabajo, ya que el acceso a la pensión de viudez se produce en igualdad de condiciones de los viudos frente a las viudas, situación que supone un paso esencial para la afirmación de los derechos fundamentales, en especial, el acceso a la pensión y la igualdad. También mencionar que tanto varones y mujeres tienen igualdad de oportunidades para acceder a dicho derecho, siendo así, no existe ninguna justificación para interpretar el artículo $53^{\circ}$ del Decreto Ley $N^{\circ} 19990$ dando mayor ventaja para el acceso a la pensión a la mujer viuda.

\section{Bibliografía}

Clark, F. (2009). Experiencia de las pensiones no contributivas en América Latina. In Envejecimiento con dignidad: pensiones no contributivas para reducir la pobreza en el Perú. Caritas, Fondo de población de las Naciones Unidas, Organización Internacional del Trabajo.

Decreto Ley N. 19990. Diario Oficial El Peruano, Lima, Perú, 30 de abril de 1973.

Gonzales Hunt, C. (2017). El Sistema Privado de Pensiones en la Jurisprudencia del TC. Gaceta Jurídica.

Morales Saravia, F. (2016). El derecho constitucional a la seguridad social y la necesidad de implementar el sistema complementario de pensiones público y privado. Vox Juris, 31(1), 5.

Neves Mujica, J. (2006). Los sistemas públicos y privados de pensiones: de la relación alternativa a la complementaria. In II Congreso Nacional de la sociedad peruana de Derecho del Trabajo y de la Seguridad Social. Sociedad Peruana de Derecho del Trabajo y de la Seguridad Social.

Sánchez-Urán Azaña, Y. (1995). Seguridad socialy Constitución. Civitas.

Vidal Bermúdez, Á. (2015). Sobre la constitucionalidad de la Ley de Reforma del Sistema Privado de Pensiones respecto de la limitación del derecho a la pensión y la libertad de contratación. Derecho PUCP: Revista de La Facultad de Derecho, 74, 521-526. 